\title{
Targeting glucose and glutamine metabolism combined with radiation therapy in non-small cell lung cancer
}

Citation for published version (APA):

Meijer, T. W. H., Peeters, W. J. M., Dubois, L. J., van Gisbergen, M. W., Biemans, R., Venhuizen, J-H., Span, P. N., \& Bussink, J. (2018). Targeting glucose and glutamine metabolism combined with radiation therapy in non-small cell lung cancer. Lung Cancer, 126, 32-40.

https://doi.org/10.1016/j.lungcan.2018.10.016

Document status and date:

Published: 01/12/2018

DOI:

10.1016/j.lungcan.2018.10.016

Document Version:

Publisher's PDF, also known as Version of record

\section{Document license:}

Taverne

Please check the document version of this publication:

- A submitted manuscript is the version of the article upon submission and before peer-review. There can be important differences between the submitted version and the official published version of record.

People interested in the research are advised to contact the author for the final version of the publication, or visit the DOI to the publisher's website.

- The final author version and the galley proof are versions of the publication after peer review.

- The final published version features the final layout of the paper including the volume, issue and page numbers.

Link to publication

\footnotetext{
General rights rights.

- You may freely distribute the URL identifying the publication in the public portal. please follow below link for the End User Agreement:

www.umlib.nl/taverne-license

Take down policy

If you believe that this document breaches copyright please contact us at:

repository@maastrichtuniversity.nl

providing details and we will investigate your claim.
}

Copyright and moral rights for the publications made accessible in the public portal are retained by the authors and/or other copyright owners and it is a condition of accessing publications that users recognise and abide by the legal requirements associated with these

- Users may download and print one copy of any publication from the public portal for the purpose of private study or research.

- You may not further distribute the material or use it for any profit-making activity or commercial gain

If the publication is distributed under the terms of Article $25 \mathrm{fa}$ of the Dutch Copyright Act, indicated by the "Taverne" license above, 


\title{
Targeting glucose and glutamine metabolism combined with radiation therapy in non-small cell lung cancer
}

\author{
Tineke W.H. Meijer ${ }^{\mathrm{a}, *}$, Wenny J.M. Peeters ${ }^{\mathrm{a}}$, Ludwig J. Dubois ${ }^{\mathrm{b}}$, Marike W. van Gisbergen ${ }^{\mathrm{b}}$, \\ Rianne Biemans ${ }^{\mathrm{b}}$, Jan-Hendrik Venhuizen ${ }^{\mathrm{c}}$, Paul N. Span ${ }^{\mathrm{a}}$, Johan Bussink ${ }^{\mathrm{a}}$

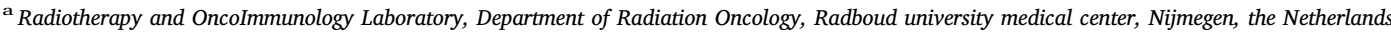 \\ ${ }^{\mathrm{b}}$ Department of Precision Medicine, The M-Lab, GROW - School for Oncology and Developmental Biology, Maastricht Comprehensive Cancer Centre, Maastricht University \\ Medical Centre, Maastricht, the Netherlands \\ ${ }^{\mathrm{c}}$ Department of Cell Biology, Radboud Institute for Molecular Life Sciences, Radboud university medical center, Nijmegen, the Netherlands
}

\section{A R T I C L E I N F O}

\section{Keywords:}

Glycolysis

Glutamine metabolism

Metabolic inhibition

Radiotherapy

Non-small cell lung cancer

\begin{abstract}
A B S T R A C T
Purpose: Metabolic inhibition might sensitize tumors to irradiation. Here, we examined the effect of lonidamine (several metabolic effects, inhibiting hexokinase amongst others) and/or 968 (glutaminase inhibitor) on tumor cell metabolism, cell growth, cytotoxicity and radiosensitivity in NSCLC cell lines in vitro in relation to histology. Materials and methods: Adeno- (H23, HCC827, H1975) and squamous cell carcinoma (H520, H292, SW900) NSCLC cells were treated with lonidamine and/or 968 for $72 \mathrm{~h}$ under physiological levels of glucose (1.5 mM). Cells were irradiated with 0,4 or $8 \mathrm{~Gy}$. Cell growth of H2B-mCherry transduced cells and cytotoxicity (CellTox ${ }^{\mathrm{TM}}$ Green Cytotoxicity Assay) were measured using live cell imaging (IncuCyte). Inhibitory effects on metabolic profiles was determined using the Seahorse XF96 extracellular Flux analyzer.

Results: NSCLC cell lines responded differently to glycolysis (lonidamine) and/or glutaminase (968) inhibition, largely corresponding with changes in glycolytic and mitochondrial metabolism upon treatment. Response patterns were not related to histology. 968 was cytotoxic in cell lines with high glutaminase C expression (H1975 and H520), whereas combination treatment was cytotoxic in KRAS mutated cell lines SW900 and H23. H292 and HCC827 were resistant to combination treatment. Treatment with 968 and especially lonidamine resulted in radiosensitization of $\mathrm{H} 292$ and HCC827 in terms of decreased relative cell growth and increased cytotoxicity. Conclusion: NSCLC is a heterogeneous disease, which is reflected in the response of different cell lines to the treatment (combinations) reported here. Only a part of NSCLC patients may benefit from the combination of radiation therapy and metabolic inhibition, making stratification necessary.
\end{abstract}

\section{Introduction}

Standard treatment for irresectable stage III non-small cell lung cancer (NSCLC) is concurrent/sequential chemoradiation. Despite this intensive therapy, prognosis remains poor with 5-year survival of 13-36\% [1]. Locoregional progression and distant metastases remain a major issue [2]. Therefore, further intensification of treatment is required.

The metabolic switch from oxidative phosphorylation of pyruvate towards glycolysis with production of lactate is involved in metastasis formation, immune escape of tumor cells, angiogenesis and radio- and chemoresistance [3-7]. Areas with residual ${ }^{18} \mathrm{~F}$-fluorodeoxyglucose $\left({ }^{18} \mathrm{~F}-\mathrm{FDG}\right)$ uptake after radiotherapy correspond with the high ${ }^{18} \mathrm{~F}$-FDG- uptake regions pre-radiotherapy in NSCLC, indicating radioresistance [8]. Glutamine metabolism of malignant cells contributes to the synthesis of ATP, lipids, amino acids and nucleotides required for proliferation, and to glutathione production for protection against oxidative stress [9]. Essential for NSCLC growth in vitro is the glutaminase 1 (GLS1) splice variant glutaminase C (GAC), an enzyme that catalyzes the hydrolysis of glutamine to glutamate, which is used in the citric acid cycle of cancer cells as a source for anaplerosis [10]. Increased expression of the glutamine transporter SLC1A5 is a poor prognostic factor in NSCLC [11]. Targeting SLC1A5 induces an increase in intracellular reactive oxygen species (ROS) and apoptosis in SLC1A5high expressing NSCLC cell lines, and attenuates NSCLC tumor growth in vivo [11,12]. Glutamine inhibition increases radiation-induced ROS

\footnotetext{
* Corresponding author at: Department of Radiation Oncology (Internal postal code 874), Radboud university medical center, P.O. Box 9101, 6500 HB Nijmegen, the Netherlands.

E-mail address: Tineke.vanZon-Meijer@radboudumc.nl (T.W.H. Meijer).
} 
and sensitizes pancreatic carcinoma to radiation in vitro and in an in vivo mice study [13]. So, inhibiting glucose and/or glutamine metabolism might optimize treatment for stage III NSCLC via depletion of energy and macromolecules as well as sensitization to chemoradiation.

Emerging evidence supports differences in metabolism between adeno- and squamous cell NSCLC, based on the expression of metabolic transporters and enzymes, ${ }^{18} \mathrm{~F}$-FDG-PET, and the level of metabolites. Squamous cell carcinomas demonstrate an anaerobic (hypoxic) glycolytic profile, i.e. poor vascularization, large necrotic areas, high lactate level together with low glucose level and elevated expression of glucose transporter 1 (GLUT1), combined with a glutaminolytic profile (increased levels of glutamate and glutathione). Adenocarcinomas have a better vascularization and likely oxygenation, and appear to have a glycolytic metabolism under aerobic conditions [11,12,14-17]. Based on these findings, it is of interest to exploit glucose and glutamine metabolism in therapies allied to NSCLC histological subtypes.

Lonidamine (1-(2,4-dichlorobenzyl)-1H-indazole-3-carboxylic acid) is an agent with several effects on cell energy metabolism. It inhibits glycolysis through interference with the function of mitochondrialbound hexokinase II, the enzyme that catalyzes the phosphorylation of glucose into glucose-6-phosphate. Furthermore, it induces lactate accumulation and thereby intracellular acidification, and it inhibits the formation of fumarate and malate. Finally, it disturbs the mitochondrial transmembrane potential, decreases the oxygen consumption rate, induces ROS through respiratory complex II (succinate dehydrogenase) inhibition, and reduces levels of NADPH and glutathione in part by inhibition of flux through the pentose phosphate pathway [18,19]. 968 (5-(3-Bromo-4-(dimethylamino)phenyl)-2,2-dimethyl-2,3,5,6-tetra-

hydrobenzo $[a]$ phenanthridin- $4(1 H)$-one) is an allosteric inhibitor, which preferentially binds to and stabilizes the inactive, monomeric state of GAC, thereby preventing it from undergoing conformational changes to the active tetramer [20,21]. If GAC reaches an activated state before 968 binding, then 968 is unable to inhibit enzyme activity. So, protein turnover is required in order to prevent the activation of GAC [20,21].

The aim of this study is to investigate the effect of treatment with lonidamine and/or 968 on metabolism, tumor cell growth, cytotoxicity and radiosensitivity in 6 NSCLC cell lines in vitro (3 adeno- and 3 squamous cell carcinoma) in relation to histology.

\section{Materials and methods}

\subsection{Cell lines}

Three adenocarcinomas (HCC827, H1975, H23) and three squamous cell carcinomas (H292, SW900, H520) were used for the experiments described below. Cell lines were authenticated by STR analysis. H1975 and HCC827 harbor an EGFR mutation (H1975: c.2369C > T (p.(Thr790Met)) (exon 20) and c.2573 T > G (p.(Leu858Arg) (exon 21); HCC827: c.2236_2250del (p.(Glu746_Ala750del)) (exon 19)). H23 and SW900 harbor a KRAS mutation. For live-cell imaging quantification of cell numbers, cell lines were labeled with nuclear localized H2BmCherry by lentiviral transduction using a pLenti6.2/V5 DEST Gateway vector (Invitrogen). Subsequently, cells were selected for blasticidin resistance, and fluorescent cells were isolated by flow cytometry. mCherry fluorescence signal diminished in H23 and H520, and could therefore not be used to determine cell count in these cell lines. All experiments were performed with RPMI medium (P04-17550, PANBiotech $\mathrm{GmbH}$, Aidenbach, Germany), supplemented with $10 \%(\mathrm{v} / \mathrm{v})$ fetal bovine serum, $1.5 \mathrm{mM}$ D-glucose and $2 \mathrm{mM}$ L-glutamine, $20 \mathrm{mM}$ Hepes, $1 \mathrm{x}$ non-essential amino acids, $20 \mathrm{U} / \mathrm{ml}$ penicillin, $20 \mathrm{ug} / \mathrm{ml}$ streptomycin (all from Gibco). To mimic the tumor microenvironment, we decided to use a physiological concentration of $1.5 \mathrm{mM}$ glucose (unless otherwise specified). A higher non-physiological glucose concentration of $10 \mathrm{mM}$ results in a significant higher glucose consumption and lactate production [22].

\subsection{Glycolysis and mitochondrial metabolism by using Seahorse}

Cells were seeded at an optimized cell density: 12.500 (H292), 20.000 (SW900), 25.000 (H1975 and HCC827) or 30.000 (H520 and $\mathrm{H} 23)$ cells/well and incubated the day after for $24 \mathrm{~h}$ with vehicle (DMSO $0.075 \%)$, lonidamide $(150 \mathrm{u} \mathrm{M}), 968(10 \mathrm{u} \mathrm{M})$ or a combination of both. Metabolic profiles were generated using the Seahorse XF96 extracellular Flux analyzer (Seahorse Bioscience) as previously described [23]. Baseline oxygen consumption rate (OCR) and extracellular rate of acidification (ECAR) were determined after replacing the growth medium with assay medium, containing the inhibitors or vehicle (without serum), according to the manufacturer's protocol [24]. A mitochondrial stress test was performed after subsequent injections of oligomycin ( $1 \mathrm{u} \mathrm{M}$; ATP synthase inhibitor), optimized FCCP $(0.2 \mathrm{u} \mathrm{M}$ for HCC827, $0.4 \mathrm{u} \mathrm{M}$ for $\mathrm{H} 23$ and $1 \mathrm{u} \mathrm{M}$ for the other cell lines) and a mixture of rotenone and antimycin A (both $1 \mathrm{u} \mathrm{M}$ ) (all from Sigma-Aldrich) to measure changes in OCR for the evaluation of mitochondrial ATP production, spare respiratory capacity and proton leak respectively. A glycolysis stress test was performed to measure changes in ECAR upon addition of glucose $(10 \mathrm{mM})$, optimized oligomycin $(1 \mathrm{u} \mathrm{M}$ for SW900 and H520, $2.5 \mathrm{u} \mathrm{M}$ for H23, HCC827 and H1975 and $5 \mathrm{u} \mathrm{M}$ for H292) and 2-deoxyglucose (2-DG, 0.1 M) (all from Sigma-Aldrich) to evaluate glycolysis and glycolytic reserve. The addition of glucose increases ECAR; this is reported as the rate of glycolysis under basal conditions. Oligomycin inhibits mitochondrial ATP production, thereby pushing cells to use glycolysis maximally. The subsequent increase in ECAR is a parameter of the cellular maximum glycolytic capacity. Finally, 2-DG is added, a glucose analog that inhibits glycolysis through competitive binding to hexokinase. The resulting decrease in ECAR confirms that the ECAR produced in the experiment is due to glycolysis. The difference between glycolytic capacity and glycolysis defines glycolytic reserve. The glycolytic reserve indicates how glycolytic a cell is, i.e. a small glycolytic reserve means that the cells are more glycolytic. Cellular respiration was corrected for total protein content, measured using the Pierce BSA protein assay kit (Thermo Fisher).

\subsection{Cell cycle}

Cells were seeded at $10 \mathrm{e} 6$ cells/well, allowed to attach overnight and afterwards incubated with inhibitors or vehicle as described above. Cell Cycle distribution was assessed by flow cytometry (FACSCanto II, BD Biosciences) after $30 \mathrm{~min}$ incubation with $10 \mu \mathrm{g} / \mathrm{ml} \mathrm{PI,} 100 \mu \mathrm{g} / \mathrm{ml}$ RNAse A and $0.1 \%$ TritonX-100 in PBS. Histograms were analyzed using FlowJo v10.0.8 (Tree Star) software and percentage cells in $\mathrm{G}_{0} /$ $\mathrm{G}_{1}, \mathrm{~S}$ or $\mathrm{G}_{2} / \mathrm{M}$-phase were calculated.

\subsection{Real time PCR}

Cells cultured in RPMI supplemented with $1.5 \mathrm{mM}$ D-glucose and $2 \mathrm{mM}$ L-glutamine were collected. Total RNA was isolated with the Norgen's Total RNA purification kit (Norgen Biotek Corp., Thorold, Ontario, Canada), and RNA $(0.5 \mu \mathrm{g})$ was reverse transcribed with the iScript cDNA synthesis kit (Bio-Rad). qPCR was performed in triple with specific primers for GLS1 (FW: 5'-AGGGTCTGTTACCTAGCTTGG-3', RV: 5'-ACGTTCGCAATCCTGTAGATTT-3' and GAC (FW: 5'-GGTCTCC TCCTCTGGATAAGATGG-3', RV: 5'-GATGTCCTCATTTGACTCAGGT GAC-3') on a CFX96 real-time PCR detection system (Bio-Rad Laboratories Inc, Richmond, CA) using SYBR Green. Levels are expressed as ratios of HPRT [25].

\subsection{Cell growth and cytotoxicity}

To assess the effect of metabolic inhibition on cell growth and cytotoxicity, cells were seeded in a 96-wells plate (\#3596, Corning; 1 column for each condition) and exposed to: control-vehicle (DMSO 0.075\%), $150 \mu \mathrm{M}$ lonidamine (Sigma-Aldrich chemie) [19], $10 \mu \mathrm{M} 968$ 
(Calbiochem) [26], combination treatment with lonidamine and 968. Dose of lonidamine was based on the data of Guo et al. showing decreased level of the cellular antioxidants glutathione and NADPH upon treatment with $150 \mu \mathrm{M}$ lonidamine [19], and dose response experiments demonstrating a decrease in confluence of about $50 \%$ for all six cell lines after $72 \mathrm{~h}$ of treatment with $150 \mu \mathrm{M}$ lonidamine (Supplementary Data 1). Decreased amounts of reducing equivalents could enhance the radiosensitivity of tumor cells and may therefore be important for radiation experiments [5]. For H292, H23, H1975 and HCC827 4000 cells were seeded; 2000 cells for SW900 and 8000 for H520. To settle, cells were left at room temperature for $20 \mathrm{~min}$. Thereafter, plates were placed in the IncuCyte ZOOM Live-Cell Analysis System (Essen BioScience) at $37^{\circ} \mathrm{C}$. Every $2 \mathrm{~h}$, images were taken of each well at $4 \mathrm{x}$ magnification for the entire experiment. When a confluence of $20-25 \%$ (i.e. $20-25 \%$ of the well occupied by tumor cells; bright-field images) was reached, cells were treated with lonidamine and/or 968, or vehicle (control), and CellTox Green (1:4000, diluted in RPMI without glucose and glutamine; Promega) was added to measure cytotoxicity. Image processing was performed using Zoom2016 A software (Essen BioScience). Images of red signal (mCherry), green signal (CellTox) and confluence were binarized for further analysis. For segmentation, thresholds were interactively set for cell lines individually at intensities where the steepest gradient occurred between background and foreground intensity levels. With these binary images, confluence, mCherry count $\mathrm{mm}^{-2}$ and cytotoxicity count $\mathrm{mm}^{-2}$ were calculated. Cytotoxicity index was calculated by cytotoxicity count $\mathrm{mm}^{-2}$ / mCherry count $\mathrm{mm}^{-2}$ (or confluence in case of unstable mCherry). Correlation coefficients of mCherry count $\mathrm{mm}^{-2}$ and confluence were 0.997-0.999 for all control cell lines with stable mCherry.

\subsection{Radiation experiments}

The same amounts of cells as described above were seeded in a 96wells plate (\#3596, Corning), and CellTox green was added. Six hours after placing 96-wells plates in the IncuCyte, cells were treated with lonidamine and/or 968. Twenty-four hours later, cells were irradiated with $0 \mathrm{~Gy}$ (control), $4 \mathrm{~Gy}$ or $8 \mathrm{~Gy}$ (XRAD 320ix, PXI precision X-ray, North Brandfod, CT). Confluence, mCherry count $\mathrm{mm}^{-2}$ and cytotoxicity count $\mathrm{mm}^{-2}$ were measured in pictures taken every $2 \mathrm{~h}$ at $4 \mathrm{x}$ magnification, and analyzed as described above. This time point of radiation was chosen based on the observation that mCherry and confluence lines are separating relative to control for both compounds after $24 \mathrm{~h}$ of treatment, reflecting inhibitory efficacy (fourth panel of Fig. 1 and Supplementary Data 2). Data from irradiated conditions were normalized to their non-irradiated counterpart to measure the radiosensitizing effect of metabolic inhibitors.

\subsection{Statistics}

Statistics were performed using Prism 4. Differences in glycolysis, mitochondrial metabolism, cell growth and cytotoxicity between treatment groups were assessed using Kruskal-Wallis test, followed by Dunn's post-hoc analyses. A p $<0.05$ was considered statistically significant.

\section{Results}

\subsection{Metabolism in 6 NSCLC cell lines}

Seahorse experiments (at $10 \mathrm{mM}$ glucose) showed that adenocarcinoma cell lines (H23, HCC827, H1975) were more glycolytic relative to squamous cell carcinoma cell lines (H520, H292, SW900) as their glycolytic reserve is smaller $(\mathrm{p}<0.0001$; Fig. 1 , Supplementary Data 2, upper panel), corresponding to earlier results of ${ }^{18} \mathrm{~F}$-FDG-PET and tissue analysis in patients which show glucose consumption under better vascularized conditions in adenocarcinomas [14,15,17]. A similar pattern, although with a lower glycolytic rate, was observed for experiments with $1.5 \mathrm{mM}$ glucose (Supplementary Data 3). H1975 showed the highest GLS1 and GAC mRNA levels (Fig. 2).

\subsection{Effect of lonidamine and/or 968 on metabolism and cell cycle}

In general, lonidamine decreased the glycolytic rate (significant for H1975, p < 0.05) (Fig. 1, Supplementary Data 2, upper panel). Baseline oxygen consumption rate (OCR) was significantly affected after treatment in $\mathrm{H} 520(\mathrm{p}=0.045)$ and $\mathrm{H} 1975(\mathrm{p}=0.034)$. 968 attenuated baseline OCR in H520 and especially H1975, corresponding with GAC mRNA levels (Fig. 1 and 2, Supplementary Data 2, second panel). 968 remarkably decreased mitochondrial ATP production in H1975, H520, HCC827 and H23. Combination treatment clearly decreased mitochondrial ATP production in all cell lines ( $\mathrm{p}<0.05$ for H520), except in H292. Mitochondrial ATP production was fully blocked in SW900 and H23. Other signs of inhibition of mitochondrial metabolism are the decrease in spare respiratory capacity and increase in proton leak, which are observed for monotherapy, and significantly for combination therapy (spare respiratory capacity: H292: $p<0.05$; proton leak: H292, H520 and H1975: p < 0.01) (Fig. 1, Supplementary Data 2 , third panel). Metabolic inhibition for $24 \mathrm{~h}$ did not affect cell cycle in any of the cell lines (Supplementary Data 4).

\subsection{Effect of lonidamine and/or 968 on cell growth and cytotoxicity}

Decrease in cell growth after treatment with lonidamine correlated partly with glycolytic rate as measured in Seahorse. HCC827 and H1975, cell lines with the highest rate of glycolysis, showed a clear response to lonidamine $72 \mathrm{~h}$ after treatment (43\% and 53\% cell growth relative to control respectively). $\mathrm{H} 292$ and $\mathrm{H} 520$, cell lines with a lower glycolytic profile, demonstrated less response to lonidamine $(60 \%$ and $67 \%$ cell growth relative to control respectively). However, glycolytic rate and response to lonidamine did not correlate for SW900 and H23 (Fig. 1, Supplementary Data 2, upper and fourth panel).

In the six cell lines, three patterns were observed after treatment with lonidamine and/or 968: decrease in cell growth without cytotoxicity, cytotoxicity due to treatment with 968 only, and a severe cytotoxic effect of the combination treatment.

H292 and HCC827 demonstrate a decrease in cell growth after (combination) treatment ( $\mathrm{p}<0.001$ for both) without an increase in cytotoxicity (Fig. 1, Supplementary Data 2, fourth and fifth panel). Three days after (combination) treatment, cells remained viable (Fig. 3, Supplementary Data 5). This corresponds with small changes in baseline OCR and mitochondrial ATP production upon (combination) treatment in H292 (Fig. 1, second and third panel).

Treatment with 968 was detrimental for the viability of H1975 cells (Fig. 4); it increased cytotoxicity significantly ( $<<0.05$ ) (Fig. 1, fourth and fifth panel). This corresponds with the high mRNA level of GAC in H1975 (Fig. 2) and decrease in baseline OCR (Fig. 1, second panel). Lonidamine decreased H1975 cell growth ( $p<0.01$ ) without a cytotoxic effect; combination treatment was very cytotoxic $(\mathrm{p}<0.001)$ (Fig. 1, fourth and fifth panel; Fig. 4). This pattern was also observed for H520, although, to a lesser extent (Supplementary Data 2, fourth and fifth panel; Supplementary Data 6).

Combination treatment was detrimental for cell growth and viability in SW900 ( $\mathrm{p}<0.001$ for both) and H23 (cell growth: $\mathrm{p}<0.001$; cytotoxicity: $\mathrm{p}<0.01$ ) (Fig. 1, Supplementary Data 2, fourth and fifth panel; Fig. 5, Supplementary Data 7). Cytotoxicity was significantly increased after combination therapy in SW900 (p $<0.001$ ), corresponding to the drastic decrease in mitochondrial metabolism in this cell line (full inhibition of mitochondrial ATP production, very high proton leak, drastic decrease in OCR; Fig. 1, second and third panel). This pattern was partly observed for $\mathrm{H} 23$ (total inhibition of mitochondrial ATP production and a very high proton leak, but no decrease in OCR) (Supplementary Data 2, second and third panel). 

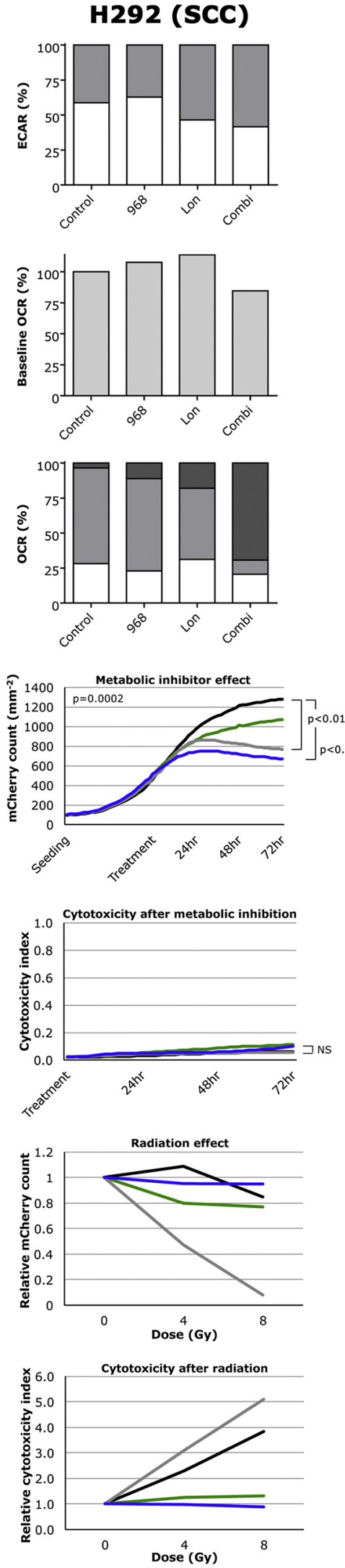

H1975 (AC)
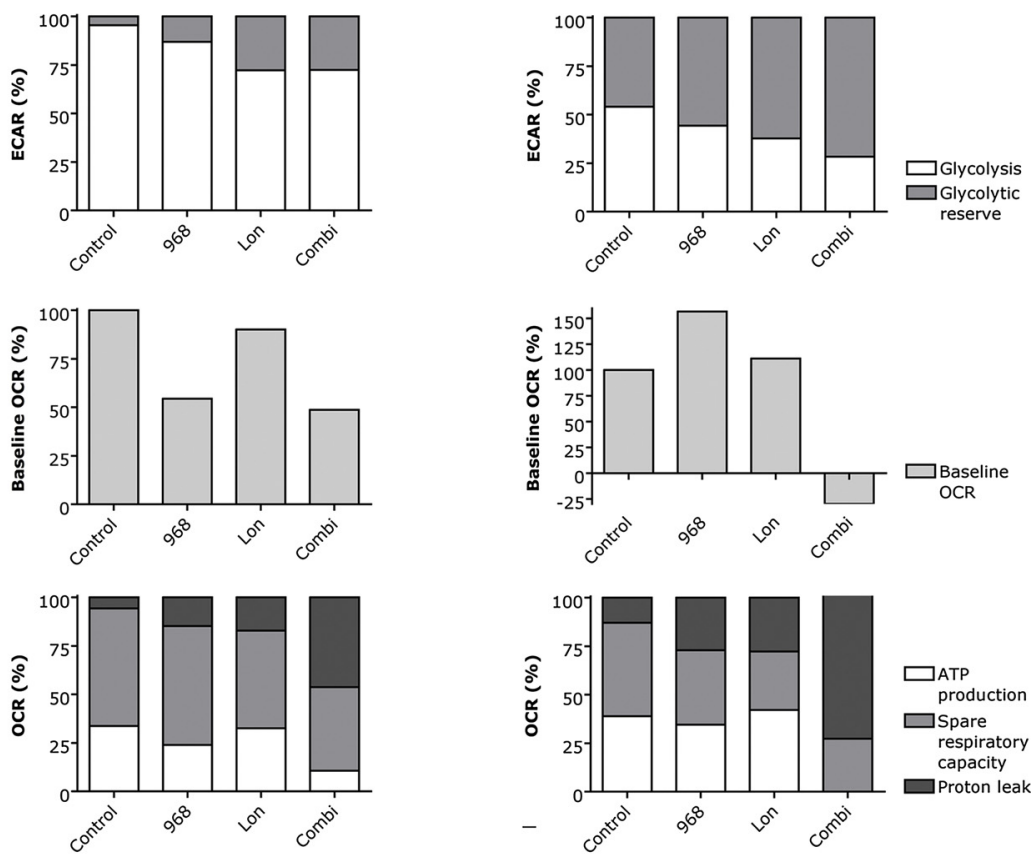

SW900 (SCC)
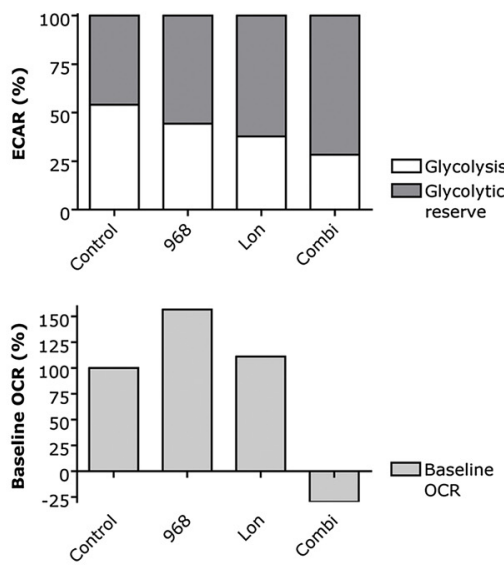
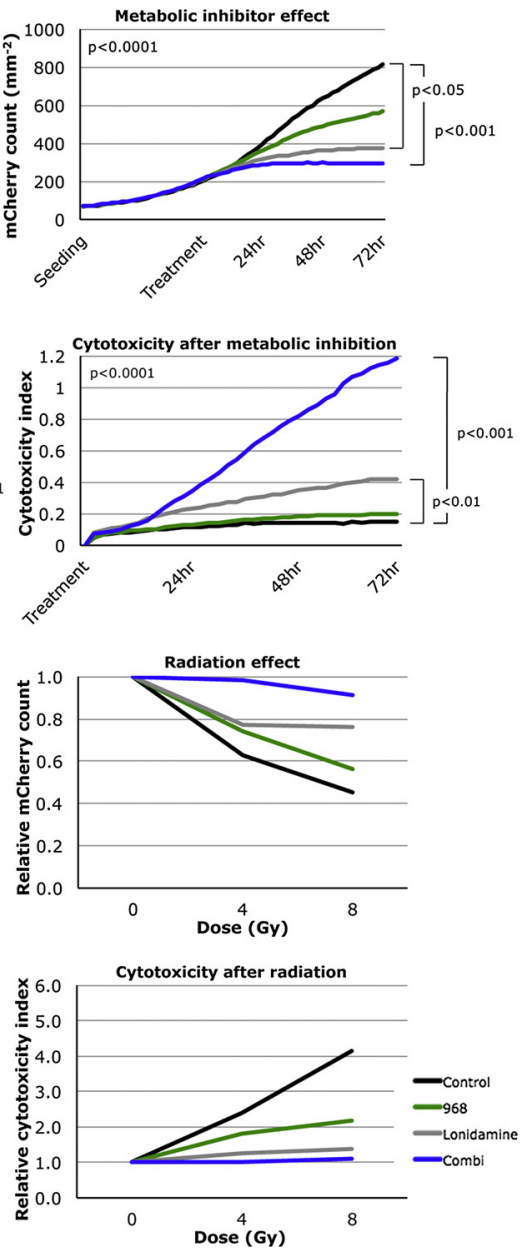
Fig. 1. Effect of metabolic inhibition on glycolysis, mitochondrial metabolism, cell growth and cytotoxicity, and radiosensitivity for H292, H1975 and SW900.

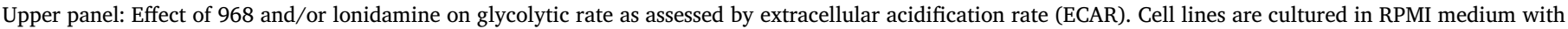
$10 \mathrm{mM}$ glucose. Bars represent mean value.

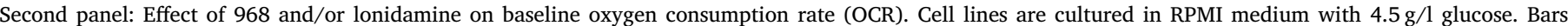
represent mean value.

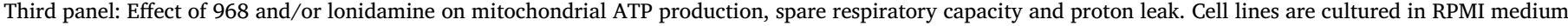
with $4.5 \mathrm{~g} / 1$ glucose. Bars represent mean value.

Fourth panel: Effect of 968 and/or lonidamine on cell growth (mCherry count

$\left(\mathrm{mm}^{-2}\right)$ ) up to three days after treatment.

Fifth panel: Effect of 968 and/or lonidamine on cytotoxicity up to three days after treatment.

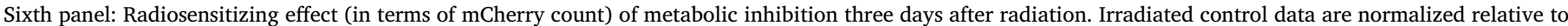
control $0 \mathrm{~Gy}$, irradiated 968 data are normalized relative to $9680 \mathrm{~Gy}$ etc. to measure the radiosensitizing effect of metabolic inhibitors.

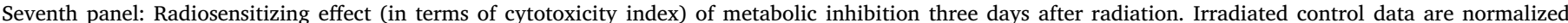
relative to control $0 \mathrm{~Gy}$, irradiated 968 data are normalized relative to $9680 \mathrm{~Gy}$ etc. to measure the radiosensitizing effect of metabolic inhibitors.

A

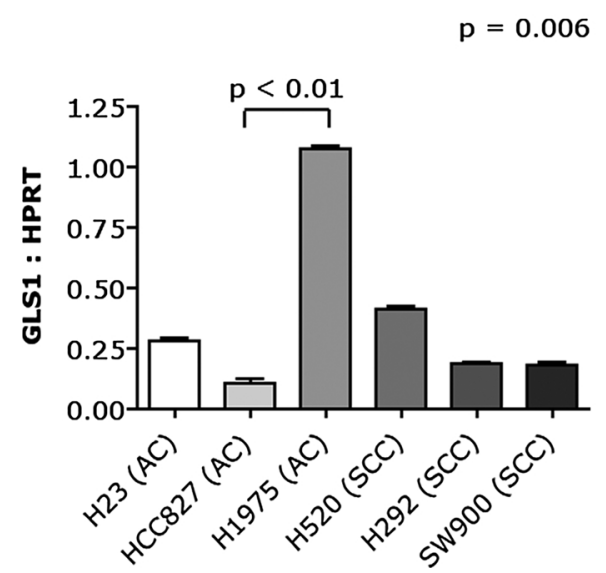

B

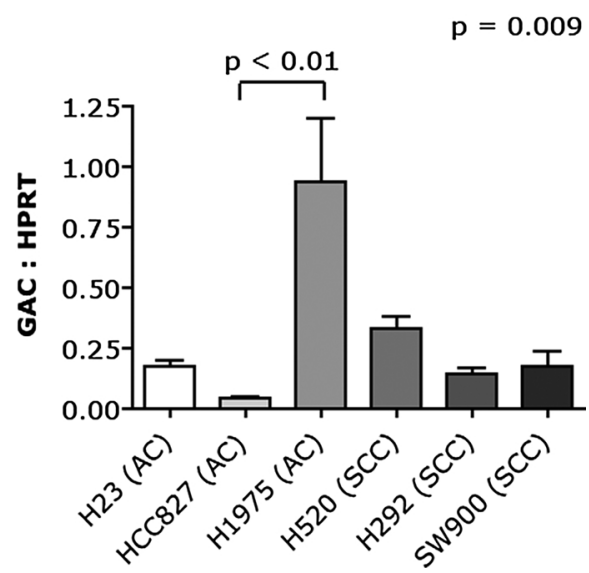

Fig. 2. mRNA levels of glutaminase in 6 NSCLC cell lines.

GLS1 mRNA level

GAC mRNA level

Cell lines are cultured in RPMI medium with $1.5 \mathrm{mM}$ glucose and $2 \mathrm{mM}$ glutamine.

Abbreviations:GACglutaminase C; GLS1: glutaminase 1. mRNA levels are expressed as ratios of HPRT.

\subsection{Radiosensitivity after metabolic inhibition}

As combination therapy was very cytotoxic for most cell lines, effect of combined metabolic inhibition on radiation response could not be assessed under these circumstances. The same applies to the combination of 968 and radiation in $\mathrm{H} 1975$ and H520.

Two SCC cell lines (H292 and H520) were remarkably resistant to radiation doses up to $8 \mathrm{~Gy}$ (decrease in cell growth of only $9-15 \%$ at 8 Gy relative to $0 \mathrm{~Gy}$ ), while the other cell lines showed a decrease in cell growth of 36-64\% (Fig. 1, Supplementary Data 2, sixth panel). Unfortunately, treatment with lonidamine or 968 did not sensitize the H520 cell line to radiation (Supplementary Data 2, sixth panel). Interestingly, the two cell lines that were resistant to (combination) metabolic inhibitors, i.e. $\mathrm{H} 292$ and HCC827, did demonstrate a radiosensitizing effect upon treatment with 968 and especially with lonidamine in terms of relative cell growth. Also, cytotoxicity index increased substantially after treatment with lonidamine and irradiation (Fig. 1, Supplementary Data 2, sixth and seventh panel; Supplementary Data 8).

A summary of results is shown in Table 1 .

\section{Discussion}

\subsection{Different response to metabolic inhibitors in NSCLC cell lines}

Earlier studies support a difference in metabolism between adenoand squamous cell NSCLC: squamous cell carcinomas demonstrate an anaerobic (hypoxic) glycolytic profile combined with glutaminolysis, while adenocarcinomas have a better vascularization/oxygenation and seem to have a glycolytic metabolism under aerobic conditions [11,12,14-17]. In this study, a varying response to metabolic inhibition was observed across different NSCLC cell lines. However, this response was not determined by histology, with both adeno- and squamous cell carcinoma cell lines exhibiting different phenotypes. So, a generalized metabolic inhibition approach for NSCLC seems not to be possible and predictive markers are required for selection of treatment.

Combination treatment of lonidamine and 968 was detrimental for cell growth and viability in the two KRAS-mutated cell lines (SW900 and H23). Monotherapy decreased cell growth, but did not affect cytotoxicity. Likely, these cell lines are able to compensate for blocking of only one of these metabolic routes. For example, glutaminolysis in the citric acid cycle can be increased upon treatment with lonidamine [19]. Furthermore, the mitochondrial isoform of phosphoenolpyruvate carboxykinase (PCK2) is expressed and active in NSCLC, including H23 $[27,28]$. PCK2 expression and activity are enhanced under low-glucose conditions. Under limited availability of glucose, PCK2 converts glutamine-derived oxaloacetate into the glycolytic intermediate phosphoenolpyruvate (PEP). Glutamine-derived PEP is used to fuel biosynthetic pathways normally sustained by glucose through gluconeogenesis (i.e. the opposite direction of glycolysis), thereby replenishing other glycolytic intermediates. These can be used in a number of biosynthetic processes, such as serine and glycerol synthesis, nucleotide synthesis and NADPH generation via the pentose phosphate pathway $[27,28]$. Also uptake of lactate may serve as a gluconeogenic 

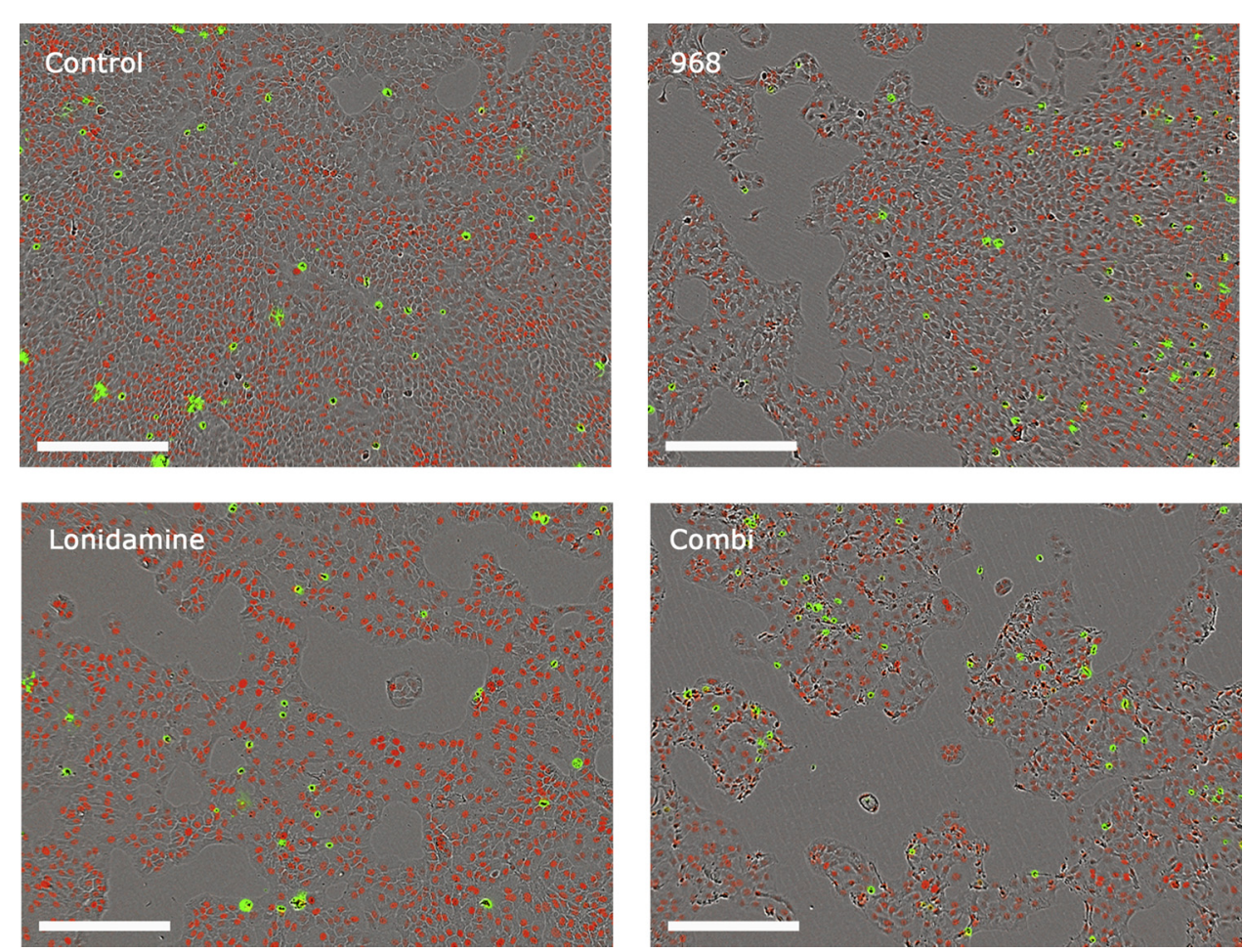

Fig. 3. Cell growth and cytotoxicity after metabolic inhibition in H292.

Microscopic images three days after metabolic inhibition for control-vehicle, 968, lonidamine and combination treatment. Magnification $4 \times$. Scale bar represents $400 \mu \mathrm{m}$. Red: mCherry, green: cytotoxicity.

precursor through PCK2 activity [27]. In this way, PCK2 activity helps to adapt to low-glucose conditions and enables glucose-independent cell proliferation. PCK2 inhibition leads to a significant increase in glucose depletion-induced apoptosis in H23 and A549 cells (both KRAS
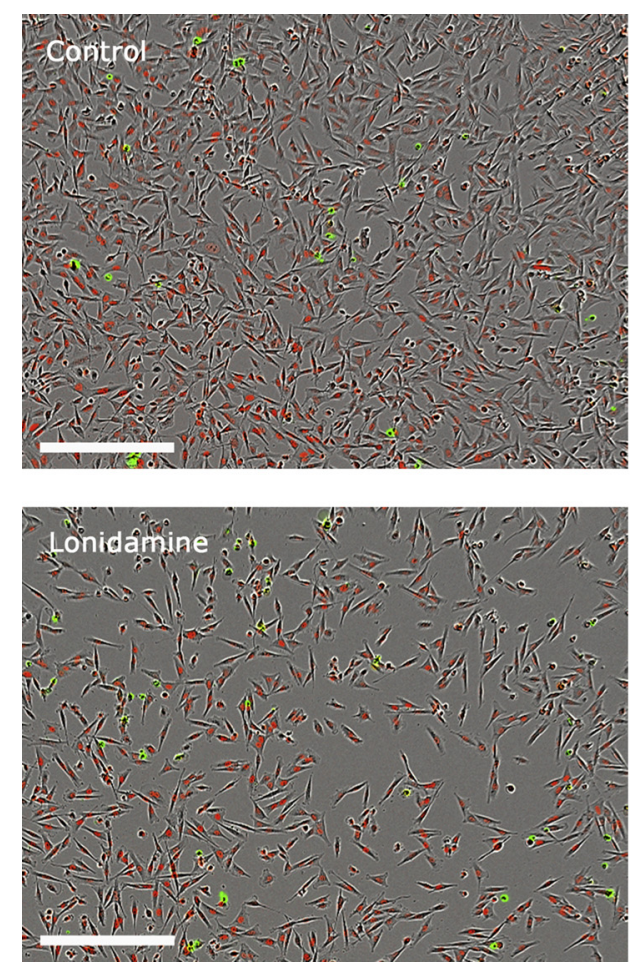

mutated) but not in H1299 cells (KRAS wildtype). Under high-glucose conditions $(20 \mathrm{mM})$, PCK2 inhibition has no effect in H23 and H549 [27]. In oncogenic KRAS- or BRAF-driven NSCLC cells, autophagy provides an intracellular glutamine supply to sustain mitochondrial
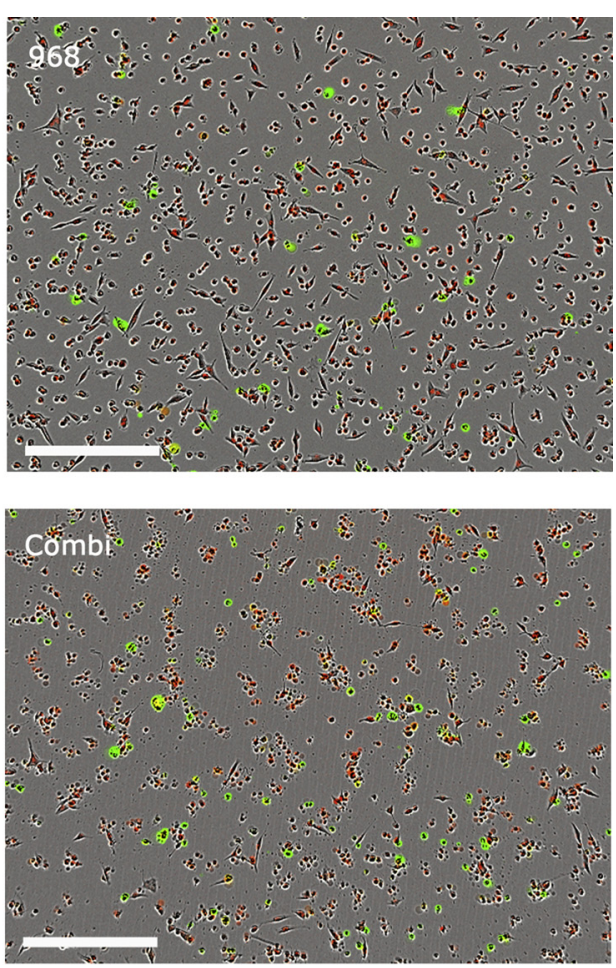

Fig. 4. Cell growth and cytotoxicity after metabolic inhibition in H1975.

Microscopic images three days after metabolic inhibition for control-vehicle, 968, lonidamine and combination treatment. Magnification $4 \times$. Scale bar represents $400 \mu \mathrm{m}$. Red: mCherry, green: cytotoxicity. 

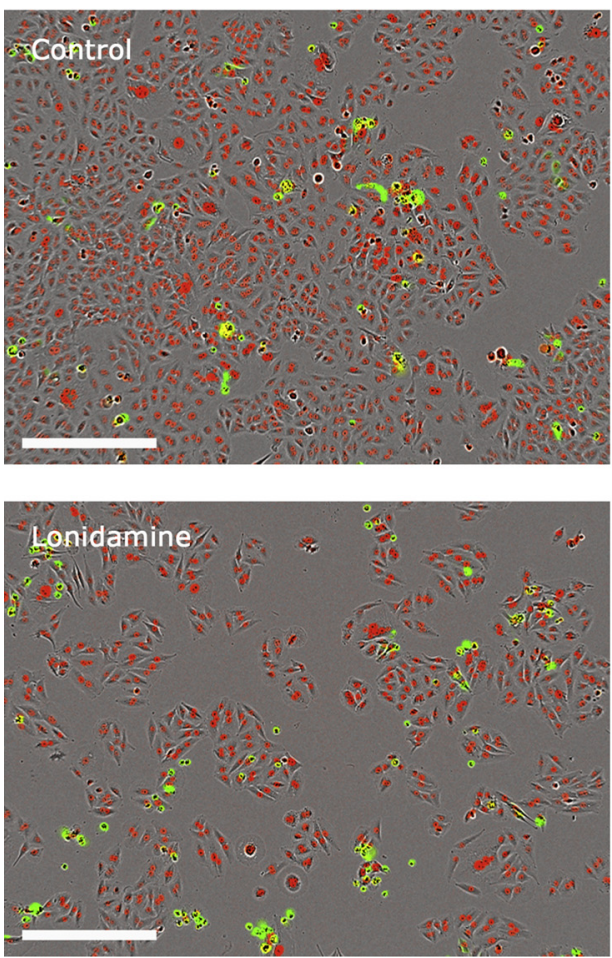
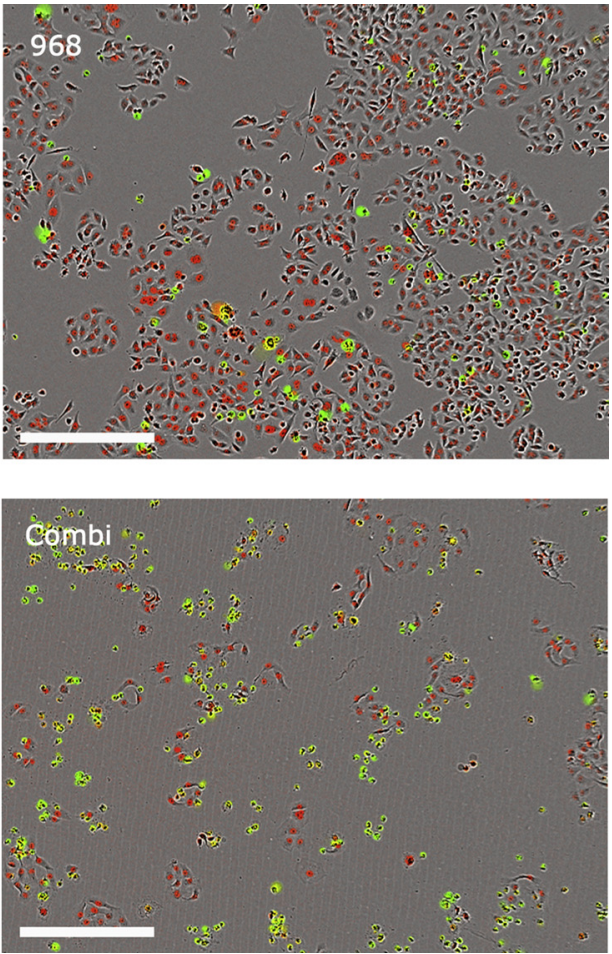

Fig. 5. Cell growth and cytotoxicity after metabolic inhibition in SW900.

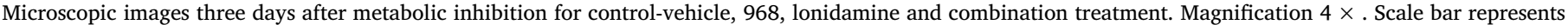
$400 \mu \mathrm{m}$. Red: mCherry, green: cytotoxicity.

Table 1

Summary of results.

\begin{tabular}{|c|c|c|c|c|c|c|}
\hline & \multicolumn{3}{|c|}{ Adenocarcinomas } & \multicolumn{3}{|c|}{ Squamous cell carcinomas } \\
\hline & $\mathrm{H} 23$ & HCC827 & H1975 & H520 & $\mathrm{H} 292$ & SW900 \\
\hline GAC mRNA & + & + & +++ & ++ & + & + \\
\hline $\begin{array}{l}\text { KRAS mutation } \\
\text { Growth reduction }\end{array}$ & Yes & No & No & No & No & Yes \\
\hline Lonidamine & + & ++ & ++ & + & ++ & ++ \\
\hline 968 & + & + & + & + & + & + \\
\hline $\begin{array}{l}\text { Combination } \\
\text { Cytotoxicity }\end{array}$ & +++ & +++ & +++ & ++ & +++ & +++ \\
\hline Lonidamine & - & - & - & - & - & + \\
\hline 968 & - & - & + & + & - & - \\
\hline $\begin{array}{l}\text { Combination } \\
\text { Radiosensitizing } \\
\text { effect }\end{array}$ & ++ & - & ++ & ++ & - & +++ \\
\hline Lonidamine & - & ++ & - & - & ++ & - \\
\hline 968 & - & + & - & - & + & - \\
\hline $\begin{array}{l}\text { Cytotoxicity after } \\
\text { radiation }\end{array}$ & & & & & & \\
\hline Lonidamine & - & + & - & - & + & - \\
\hline 968 & - & - & - & - & - & - \\
\hline
\end{tabular}

function [29]. Moreover, reprogramming of glutamine metabolism mediated by oncogenic KRAS in pancreatic ductal adenocarcinoma cells results in the conversion of glutamine-derived aspartate into pyruvate through malic enzyme 1 with the production of NADPH for maintaining redox homeostasis [30,31]. RAS-driven cancer cells display aerobic glycolysis and glutamine can provide carbon for anaplerosis in these cells $[9,32,33]$. So, KRAS mutation might be a predictive marker for response to combination inhibition of glycolysis and glutamine metabolism.

NSCLC cell lines show a variable range of glutamine dependency [10]. Glutaminase inhibition by 968 was cytotoxic for cell lines with high GAC mRNA level (H1975 and H520). Therefore, GAC expression could be a predictive marker for response to 968 monotherapy. H292 and HCC827 demonstrated a decrease in cell growth after (combination) treatment without an increase in cytotoxicity. Apparently, these cell lines are able to compensate for this (combination) therapy. Remarkably, H292 showed only a small decrease in baseline OCR and had the highest mitochondrial ATP production after combination treatment (Fig. 1, second and third panel; Fig. 3), possibly due to metabolism of other fuels like lipids and lactate instead of glucose and glutamine [34-36]. Recently, it was demonstrated that lactate-derived carbons are detected in citric acid cycle intermediates in tumors derived from HCC827 cell line [35]. Furthermore, HCC827 and H292 showed a substantial increase in basal respiration when exposed to glucose-free medium containing fatty acids relative to medium containing glucose, while this increase was not observed for H1975 (preliminary unpublished data). This observation further strengthens our hypothesis that H292 and HCC827 cells are able to survive inhibition of glucose and glutamine metabolism by fueling fatty acids in mitochondrial respiration.

Goodwin et al. performed glycolysis inhibition experiments in adeno- (A549 and H522) and squamous cell (HCC95 and HCC1588) NSCLC cell lines by using GLUT1 knockdown, 2-deoxyglucose and WZB117 (a selective, small-molecule GLUT1 inhibitor) [37]. GLUT1 knockdown decreased glucose uptake, markedly suppressed the proliferative capacity of the squamous cell carcinoma cell lines HCC95 and HCC1588, accompanied by extensive apoptosis and cell death, and lower amount of intracellular ATP and NAD(P)H. GLUT1 knockdown also decreased glucose uptake in adenocarcinoma cell lines A549 and H522. In contrast to squamous cell carcinoma cell lines, these adenocarcinoma cell lines showed only moderately suppressed proliferation upon GLUT1 knockdown without induction of apoptosis or cell death and no changes in intracellular amount of ATP [37]. GLUT1 knockdown significantly inhibited in vivo growth of HCC95 tumors, but did not affect in vivo growth of $\mathrm{H} 549$ and H522 tumors. Squamous cell carcinoma cell lines HCC95 and HCC1588 were more apoptotic upon treatment with 2-deoxyglucose or WZB117 compared to A549 and H522 adenocarcinoma cell lines, despite similar decrease in glucose 
uptake and extracellular acidification rate. 2-Deoxyglycose-treated or WZB117-treated HCC1588 squamous cell carcinoma tumors exhibited significant in vivo tumor growth reduction, with no anti-tumor activity in treated A549 adenocarcinomas [37].

This selective cytotoxicity of glycolysis inhibition in squamous cell carcinomas observed by Goodwin et al. suggests a specific reliance on glucose metabolism in squamous cell carcinomas and metabolic compensatory mechanism to maintain bioenergetics, cellular viability and proliferative capacity in adenocarcinomas. We also found metabolic flexibility among NSCLC cell lines, but we could not support this histology-based difference in our in vitro experiments, possibly due to the fact that lonidamine has multiple effects on metabolism apart from glycolysis inhibition. Furthermore, heterogeneity is likely to exist within these histological subtypes. For example, the A549 adenocarcinoma cell line was more sensitive to GLUT1 knockdown compared to H522 adenocarcinoma cell line, in agreement with higher GLUT1 mRNA and protein expression in A549. Moreover, in adenocarcinoma patient tumors, predominantly micropapillary and solid adenocarcinomas exhibit higher GLUT1 expression and ${ }^{18} \mathrm{~F}$-FDG-uptake compared to adenocarcinomas with a predominantly lepidic, acinar or papillary growth pattern [38,39], further supporting heterogeneity among subclassifications. Heterogeneity might also exist among squamous cell carcinoma cell lines, as the HCC2450 squamous cell carcinoma tumor line exhibits much lower GLUT1 mRNA and protein compared to the examined HCC1588 and HCC95 cell lines. Unfortunately, HCC2450 was not exposed to GLUT1 knockdown, 2-deoxyglucose or WZB117 in the study by Goodwin et al [37].

An interesting observation in our study is the increase in proton leak for both compounds, but especially for combination treatment in all cell lines. Proton leak gives an indication about the leakage of protons out of the mitochondrial space. Oxidative phosphorylation runs according to the following principles: i) Electrons of the tricarboxylic acid cycle donate their energy in a series of redox reactions, in which oxygen is reduced into water; ii) This energy is used to create a proton gradient; iii) This proton gradient drives ATP synthesis. So, compounds disturbing the mitochondrial transmembrane structure or potential could influence proton leak. Lonidamine is known to disturb the mitochondrial transmembrane potential [18], and thus is likely to influence proton leak. For 968, this effect has not been described.

\subsection{Metabolic inhibition and response to radiation in NSCLC}

Lonidamine and 968 demonstrated a radiosensitizing effect in $\mathrm{H} 292$ and HCC827. Sappington et al. observed that BPTES, a GLS-1 specific inhibitor, reduced cell viability and proliferation and substantially reduced extracellular glutathione excretion in H460 and H549 cells. GLS1 inhibition markedly resulted in a radiosensitizing effect in these cell lines, suggesting the importance of glutamine-derived glutathione in redox control and radioresistance [40]. A key metabolic role of oncogenic KRAS is to maintain redox balance, by regulating metabolism of glutamine amongst others [41]. Glutamine deprivation sensitized the KRAS mutated NSCLC cell line HCC44 to radiation, but not the KRAS wildtype H522 [30]. We could not confirm the radiosensitizing effect of glutamine inhibition in KRAS mutated cell lines. The effect of glutaminase inhibition on the redox status (higher ROS, and decrease in glutathione and NADPH) and radiosensitivity has also been observed for cervical and pancreatic cancer cells $[13,42]$. Also lonidamine induces ROS formation and inhibits NADPH and glutathione generation [19]. However, in four NSCLC cell lines, we did not observe a radiosensitizing effect of metabolic inhibitors. Conversely, metabolic inhibition resulted in radioresistance in H1975, SW900, H23 an H292. We hypothesized that metabolic inhibition resulted in cell cycle arrest due to depletion of energy and macromolecules, as arrest in G0/G1 phase causes radioresistance, but we were unable to confirm this hypothesis. Han et al. showed that 968 inhibited NSCLC cell proliferation and arrested G0/G1 phase of cell cycle in A549 and H23 [43]. These different observations between studies might be due to the heterogeneity of NSCLC cell lines and differences in experimental set-up, like glucose concentration and time of treatment with metabolic inhibitor. Furthermore, 968 and combined treatment could be too cytotoxic to be able to demonstrate a radiosensitizing effect in H1975, SW900, H23 and H292 (Fig. 4-5, Supplementary Data 6-7). A lower compound dose, probably resulting in less cytotoxicity, may increase the radiation effect.

The diversity in response of NSCLC cell lines to the combination of metabolic inhibition and radiation therapy may explain the negative result of the prospective randomized comparison of radiation therapy plus lonidamine versus radiation therapy alone for nonresectable NSCLC [44]. A trend towards better local control was observed for patients treated with lonidamine and radiation, which suggests that only a part of patients benefit from treatment with lonidamine, underlining the need to better select patients for such treatment modifications [44]. Thus, a general approach regarding metabolic inhibition to improve radiotherapy efficacy is not possible for NSCLC and predictive markers are required.

\subsection{Translation of in vitro results into in vivo}

The tumor microenvironment can affect cancer cell metabolism [34]. For this reason, we mimicked the tumor microenvironment by using a more physiological concentration of $1.5 \mathrm{mM}$ glucose [22]. Glucose concentrations vary within tumors, and many tumors have average levels between 1 and $2 \mathrm{mM}$. However, other nutrients like glutamine are also in excess in standard culture medium and might also have an impact on our results. Furthermore, in standard culture conditions, oxygen is abundant (21\%), while oxygen levels in even the best perfused tissues are $9 \%$ or less and can be very low in tumors [34]. As oxygen level influences metabolism [45], this could have an impact on the response to metabolic inhibitors in vivo. Moreover, human NSCLC tumors demonstrate metabolically heterogeneous regions within tumors related to the degree of perfusion [46]. All together, this implies that the tumor microenvironment, including tumor vasculature supplying nutrients and oxygen, influences the metabolic phenotype of cancer cells and sensitivity to metabolic inhibitors.

\subsection{Conclusion}

NSCLC is a heterogeneous set of diseases. Cell lines responded differently to glycolysis and/or glutaminase inhibition, largely corresponding with changes in glycolytic and mitochondrial metabolism upon treatment, but response patterns were not related to histological subtype. Interestingly, response patterns were associated with KRAS mutation status and level of GAC expression. The glutaminase inhibitor 968 decreased cell growth and was cytotoxic in cell lines with high GAC expression (H1975 and H520), whereas combination treatment was detrimental for cell growth and viability in the KRAS mutated cell lines (SW900 and H23). Two of six cell lines (H292 and HCC827) were quite resistant to combination treatment and may depend on or switch to other fuels like phospholipids and lactate. 968 and especially lonidamine were able to radiosensitize the H292 and HCC827 NSCLC cell lines. Therefore, only a part of NSCLC patients may benefit from the combination of radiation therapy and metabolic inhibition, making stratification and predictive markers necessary. As the tumor microenvironment affects the metabolic phenotype of tumor cells, these observations should be confirmed in in vivo models and patients.

\section{Conflict of interest}

The authors declare no potential conflicts of interest. 


\section{Financial support}

\section{We did not receive financial support.}

\section{Appendix A. Supplementary data}

Supplementary material related to this article can be found, in the online version, at doi:https://doi.org/10.1016/j.lungcan.2018.10.016.

\section{References}

[1] P. Goldstraw, K. Chansky, J. Crowley, R. Rami-Porta, H. Asamura, W.E. Eberhardt, et al., The IASLC Lung Cancer Staging Project: Proposals for Revision of the TNM Stage Groupings in the Forthcoming (Eighth) Edition of the TNM Classification for Lung Cancer, J. Thorac. Oncol. 11 (2016) 39-51.

[2] A. Auperin, C. Le Pechoux, E. Rolland, W.J. Curran, K. Furuse, P. Fournel, et al., Meta-analysis of concomitant versus sequential radiochemotherapy in locally advanced non-small-cell lung cancer, J. Clin. Oncol. 28 (2010) 2181-2190.

[3] P. Danhier, C.J. De Saedeleer, O. Karroum, G. De Preter, P.E. Porporato, B.F. Jordan, et al., Optimization of tumor radiotherapy with modulators of cell metabolism: toward clinical applications, Semin. Radiat. Oncol. 23 (2013) 262-272.

[4] F. Hirschhaeuser, U.G. Sattler, W. Mueller-Klieser, Lactate, A metabolic key player in cancer, Cancer Res. 71 (2011) 6921-6925.

[5] T.W. Meijer, J.H. Kaanders, P.N. Span, J. Bussink, Targeting hypoxia, HIF-1, and tumor glucose metabolism to improve radiotherapy efficacy, Clin. Cancer Res. 18 (2012) 5585-5594.

[6] U.G. Sattler, W. Mueller-Klieser, The anti-oxidant capacity of tumour glycolysis, Int. J. Radiat. Biol. 85 (2009) 963-971.

[7] S. Ganapathy-Kanniappan, J.F. Geschwind, Tumor glycolysis as a target for cancer therapy: progress and prospects, Mol. Cancer 12 (2013) 152.

[8] H.J. Aerts, J. Bussink, W.J. Oyen, W. van Elmpt, A.M. Folgering, D. Emans, et al., Identification of residual metabolic-active areas within NSCLC tumours using a preradiotherapy FDG-PET-CT scan: a prospective validation, Lung Cancer 75 (2012) 73-76.

[9] S.Y. Lunt, Vander Heiden, MG Aerobic glycolysis: meeting the metabolic requirements of cell proliferation, Annu. Rev. Cell Dev. Biol. 27 (2011) 441-464.

[10] A.P. van den Heuvel, J. Jing, R.F. Wooster, K.E. Bachman, Analysis of glutamine dependency in non-small cell lung cancer: GLS1 splice variant GAC is essential for cancer cell growth, Cancer Biol. Ther. 13 (2012) 1185-1194.

[11] M. Hassanein, J. Qian, M.D. Hoeksema, J. Wang, M. Jacobovitz, X. Ji, et al., Targeting SLC1a5-mediated glutamine dependence in non-small cell lung cancer, Int. J. Cancer 137 (2015) 1587-1597.

[12] M. Hassanein, M.D. Hoeksema, M. Shiota, J. Qian, B.K. Harris, H. Chen, et al., SLC1A5 mediates glutamine transport required for lung cancer cell growth and survival, Clin. Cancer Res. 19 (2013) 560-570.

[13] D. Li, Z. Fu, R. Chen, X. Zhao, Y. Zhou, B. Zeng, et al., Inhibition of glutamine metabolism counteracts pancreatic cancer stem cell features and sensitizes cells to radiotherapy, Oncotarget 6 (2015) 31151-31163.

[14] T.W. Meijer, O.C. Schuurbiers, J.H. Kaanders, M.G. Looijen-Salamon, L.F. de GeusOei, A.F. Verhagen, et al., Differences in metabolism between adeno- and squamous cell non-small cell lung carcinomas: spatial distribution and prognostic value of GLUT1 and MCT4, Lung Cancer 76 (2012) 316-323.

[15] O.C. Schuurbiers, T.W. Meijer, J.H. Kaanders, M.G. Looijen-Salamon, L.F. de GeusOei, M.A. van der Drift, et al., Glucose metabolism in NSCLC is histology-specific and diverges the prognostic potential of 18FDG-PET for adenocarcinoma and squamous cell carcinoma, J. Thorac. Oncol. 9 (2014) 1485-1493.

[16] C.M. Rocha, A.S. Barros, B.J. Goodfellow, I.M. Carreira, A. Gomes, V. Sousa, et al., NMR metabolomics of human lung tumours reveals distinct metabolic signatures for adenocarcinoma and squamous cell carcinoma, Carcinogenesis 36 (2015) 68-75.

[17] T.W. Meijer, L.F. de Geus-Oei, E.P. Visser, W.J. Oyen, M.G. Looijen-Salamon, D. Visvikis, et al., Tumor Delineation and Quantitative Assessment of Glucose Metabolic Rate within Histologic Subtypes of Non-Small Cell Lung Cancer by Using Dynamic 18F Fluorodeoxyglucose PET, Radiology (2016) 160329.

[18] L. Ravagnan, I. Marzo, P. Costantini, S.A. Susin, N. Zamzami, P.X. Petit, et al., Lonidamine triggers apoptosis via a direct, Bcl-2-inhibited effect on the mitochondrial permeability transition pore, Oncogene 18 (1999) 2537-2546.

[19] L. Guo, A.A. Shestov, A.J. Worth, K. Nath, D.S. Nelson, D.B. Leeper, et al., Inhibition of Mitochondrial Complex II by the Anticancer Agent Lonidamine, J. Biol. Chem. 291 (2016) 42-57.

[20] K.F. Wilson, J.W. Erickson, M.A. Antonyak, Cerione RA Rho GTPases and their roles in cancer metabolism, Trends Mol. Med. 19 (2013) 74-82.

[21] C.A. Stalnecker, S.M. Ulrich, Y. Li, S. Ramachandran, M.K. McBrayer,
R.J. DeBerardinis, et al., Mechanism by which a recently discovered allosteric inhibitor blocks glutamine metabolism in transformed cells, Proc Natl Acad Sci U S A 112 (2015) 394-399.

[22] K. Birsoy, R. Possemato, F.K. Lorbeer, E.C. Bayraktar, P. Thiru, B. Yucel, et al., Metabolic determinants of cancer cell sensitivity to glucose limitation and biguanides, Nature 508 (2014) 108- +..

[23] M.W. van Gisbergen, A.M. Voets, R. Biemans, R.F. Hoffmann, M.J. Drittij-Reijnders, G. Haenen, et al., Distinct radiation responses after in vitro mtDNA depletion are potentially related to oxidative stress, PLoS One 12 (2017) e0182508.

[24] D.G. Nicholls, V.M. Darley-Usmar, M. Wu, P.B. Jensen, G.W. Rogers, D.A. Ferrick, Bioenergetic profile experiment using C2C12 myoblast cells, J. Vis. Exp. (2010).

[25] J.B. de Kok, R.W. Roelofs, B.A. Giesendorf, J.L. Pennings, E.T. Waas, T. Feuth, et al., Normalization of gene expression measurements in tumor tissues: comparison of 13 endogenous control genes, Lab. Invest. 85 (2005) 154-159.

[26] J.B. Wang, J.W. Erickson, R. Fuji, S. Ramachandran, P. Gao, R. Dinavahi, et al., Targeting mitochondrial glutaminase activity inhibits oncogenic transformation, Cancer Cell 18 (2010) 207-219.

[27] K. Leithner, A. Hrzenjak, M. Trotzmuller, T. Moustafa, H.C. Kofeler, C. Wohlkoenig, et al., PCK2 activation mediates an adaptive response to glucose depletion in lung cancer, Oncogene 34 (2015) 1044-1050.

[28] E.E. Vincent, A. Sergushichev, T. Griss, M.C. Gingras, B. Samborska, T. Ntimbane, et al., Mitochondrial Phosphoenolpyruvate Carboxykinase Regulates Metabolic Adaptation and Enables Glucose-Independent Tumor Growth, Mol. Cell 60 (2015) 195-207.

[29] R.J. DeBerardinis, Chandel NS Fundamentals of cancer metabolism, Sci. Adv. 2 (2016) e1600200.

[30] G. Chakrabarti, Mutant, KRAS associated malic enzyme 1 expression is a predictive marker for radiation therapy response in non-small cell lung cancer, Radiat. Oncol. 10 (2015) 145.

[31] J. Son, C.A. Lyssiotis, H. Ying, X. Wang, S. Hua, M. Ligorio, et al., Glutamine supports pancreatic cancer growth through a KRAS-regulated metabolic pathway, Nature 496 (2013) 101-105.

[32] P.S. Ward, C.B. Thompson, Metabolic reprogramming: a cancer hallmark even warburg did not anticipate, Cancer Cell 21 (2012) 297-308.

[33] S.M. Davidson, T. Papagiannakopoulos, B.A. Olenchock, J.E. Heyman, M.A. Keibler, A. Luengo, et al., Environment Impacts the Metabolic Dependencies of Ras-Driven Non-Small Cell Lung Cancer, Cell Metab. 23 (2016) 517-528.

[34] Vander Heiden MG, DeBerardinis RJ Understanding the Intersections between Metabolism and Cancer Biology, Cell 168 (2017) 657-669.

[35] B. Faubert, K.Y. Li, L. Cai, C.T. Hensley, J. Kim, L.G. Zacharias, et al., Lactate metabolism in human lung tumors, Cell 171 (2017) 358-371 e359.

[36] C. Corbet, A. Pinto, R. Martherus, J.P. Santiago de Jesus, F. Polet, O. Feron, Acidosis Drives the Reprogramming of Fatty Acid Metabolism in Cancer Cells through Changes in Mitochondrial and Histone Acetylation, Cell Metab. 24 (2016) 311-323.

[37] J. Goodwin, M.L. Neugent, S.Y. Lee, J.H. Choe, H. Choi, D.M.R. Jenkins, et al., The distinct metabolic phenotype of lung squamous cell carcinoma defines selective vulnerability to glycolytic inhibition, Nat. Commun. 8 (2017) 15503.

[38] K. Kadota, C. Colovos, K. Suzuki, N.P. Rizk, M.P. Dunphy, E.C. Zabor, et al., FDG PET SUVmax Combined with IASLC/ATS/ERS Histologic Classification Improves the Prognostic Stratification of Patients with Stage I Lung Adenocarcinoma, Ann. Surg. Oncol. 19 (2012) 3598-3605.

[39] H. Nakamura, H. Saji, T. Shinmyo, R. Tagaya, N. Kurimoto, H. Koizumi, et al., Close association of IASLC/ATS/ERS lung adenocarcinoma subtypes with glucose-uptake in positron emission tomography, Lung Cancer 87 (2015) 28-33.

[40] D.R. Sappington, E.R. Siegel, G. Hiatt, A. Desai, R.B. Penney, A. Jamshidi-Parsian, et al., Glutamine drives glutathione synthesis and contributes to radiation sensitivity of A549 and H460 lung cancer cell lines, Biochim. Biophys. Acta 1860 (2016) 836-843.

[41] A.C. Kimmelman, Metabolic, Dependencies in RAS-Driven cancers, Clin. Cancer Res. 21 (2015) 1828-1834.

[42] L. Xiang, G. Xie, C. Liu, J. Zhou, J. Chen, S. Yu, et al., Knock-down of glutaminase 2 expression decreases glutathione, $\mathrm{NADH}$, and sensitizes cervical cancer to ionizing radiation, Biochim. Biophys. Acta 1833 (2013) 2996-3005.

[43] T. Han, M. Guo, T. Zhang, M. Gan, C. Xie, J.B. Wang, A novel glutaminase inhibitor968 inhibits the migration and proliferation of non-small cell lung cancer cells by targeting EGFR/ERK signaling pathway, Oncotarget 8 (2017) 28063-28073.

[44] C.W. Scarantino, A.J. McCunniff, G. Evans, C.W. Young, Paggiarino DA A prospective randomized comparison of radiation therapy plus lonidamine versus radiation therapy plus placebo as initial treatment of clinically localized but nonresectable nonsmall cell lung cancer, Int. J. Radiat. Oncol. Biol. Phys. 29 (1994) 999-1004.

[45] S.E. Rademakers, P.N. Span, J.H. Kaanders, F.C. Sweep, A.J. van der Kogel, J. Bussink, Molecular aspects of tumour hypoxia, Mol. Oncol. 2 (2008) 41-53.

[46] C.T. Hensley, B. Faubert, Q. Yuan, N. Lev-Cohain, E. Jin, J. Kim, et al., Metabolic heterogeneity in human lung tumors, Cell 164 (2016) 681-694. 